\title{
X-Ray Crystallography and its Role in Understanding the Physicochemical Properties of Pharmaceutical Cocrystals
}

\author{
Srinivasulu Aitipamula ${ }^{1^{*}}$ and Venu R. Vangala ${ }^{2^{*}}$
}

\begin{abstract}
Properties of a matter are intrinsically dependent on the internal arrangement of molecules in the solid state. Therefore, knowledge of three-dimensional structure of the matter is a prerequisite for structure-property correlations and design of functional materials. Over the past century, X-ray crystallography has evolved as a method of choice for accurate determination of molecular structure at atomic resolution. The structural information obtained from crystallographic analysis paved the way for rapid development in electronic devices, mineralogy, geosciences, materials science, pharmaceuticals, etc. Knowledge of the structural information of active pharmaceutical ingredients (APIs) is a prerequisite for rational drug design and synthesis of new chemical entities for development as new medicines. Over the past two decades, X-ray crystallography has played a key role in the design of pharmaceutical cocrystals_crystalline solids containing an API and one or more of pharmaceutically acceptable coformers. These materials have proved promising for fine-tuning several important properties of APIs. This short review highlights the history of crystallography, early breakthroughs, and the role of crystallography in understanding the physicochemical properties of pharmaceutical cocrystals.
\end{abstract}

\section{Introduction}

Any matter on the earth is made up of atoms connected by interatomic bonds. The precise nature of the interatomic bonds determines the properties of the matter. Curiosity over understanding the properties of a material led to investigate how in different ways the atoms are arranged to form a certain molecule and intermolecular bonds that connect the molecules to form the solid. In this regard, X-ray crystallography is undoubtedly the most comprehensive technique available to determine the atomic positions, intermolecular interactions, and the overall structure of any molecule at atomic resolution. ${ }^{1}$ From the first use for determination of crystal structure of $\mathrm{NaCl}, \mathrm{X}$-ray diffraction has received paramount importance in many fields of science for understanding the structure-property correlations. ${ }^{2}$ For example, the fact that the hardness of diamond and the brittleness of graphite are due to the different ways that the carbon atoms are arranged in the crystal lattice would not have been revealed unless their crystal structures were determined by X-ray diffraction.

Over the past 100 years, diffraction-based crystallography has become a mainstream and powerful experimental tool to determine the three-dimensional structure of small and macromolecules. ${ }^{3}$ Many pioneering works have led to the crystallography to a state where it stands today as a mature discipline of far-reaching impact on material characterization and analysis. The emergence of X-ray crystallography ensued greater understanding of the properties
X-ray crystallography: An analytical technique used for determining the atomic and molecular structure of a crystal, in which the crystalline atoms cause a beam of incident $\mathrm{X}$-rays to diffract into many specific directions.

${ }^{\star}$ Correspondence: srinivasulu_aitipamula@ ices.a-star.edu.sg; V.G.R.Vangala@bradford. ac.uk

${ }^{1}$ Crystallization and Particle Sciences, Institute of Chemical and Engineering Sciences, $A^{*}$ STAR (Agency for Science, Technology and Research), 1 Pesek Road, Jurong Island, Singapore 627833, Singapore

${ }^{2}$ Centre for Pharmaceutical Engineering Science, School of Pharmacy and Medical Sciences, University of Bradford, Bradford BD7 1DP, UK 
APIs: According to US Food and Drug Administration (US-FDA), an API is any substance or mixture of substances intended to be used in the manufacture of a drug product and that, when used in the production of a drug, becomes an active ingredient in the drug product. Such substances are intended to furnish pharmacological activity or other direct effect in the diagnosis, cure, mitigation, treatment, or prevention of disease or to affect the structure and function of the body.

X-rays: Electromagnetic radiation of wavelength $0.1-100$

$\AA$, produced by bombarding a target (generally a metal such as $\mathrm{Cu}$ or $\mathrm{Mo}$ ) with fast electrons.

Bragg's law : It refers to the simple equation: $n \lambda=2 d$ $\sin \theta$ which explains why

the cleavage faces of crystals appear to reflect X-ray beams at certain angles of incidence $(\theta, \lambda)$, where $d$ is the distance between atomic layers in a crystal and $\lambda$ is the wavelength

of the incident X-ray beam.

When X-rays strike a crystal, they will be diffracted only when this equation is satisfied. of materials and functioning of biological molecules. The structural insights obtained from crystallographic analysis led to unprecedented developments in electronic devices, mineralogy, geosciences, materials science, and pharmaceuticals. Knowledge of accurate structural information of active pharmaceutical ingredients (APIs) is a prerequisite for rational drug design and synthesis of new chemical entities for the development of new medicines. ${ }^{4}$ With a focus on the role of crystallography in modern drug development, this review is an attempt to highlight the brief history of crystallography, early breakthroughs, and the role of crystallography in understanding the physicochemical properties of pharmaceutical cocrystals.

\subsection{History of X-ray Crystallography}

The discovery and development of X-ray crystallography as a tool for precise analysis of solidstate matter is the result of many pioneering works. X-rays were discovered by W. C. Röentgen in $1895 .^{5}$ The ability of X-rays to be diffracted by single crystals was demonstrated by $\mathrm{M}$. von Laue in 1912, which earned him the Nobel Prize for physics in $1914 .{ }^{6}$ In the subsequent year, W.L. Bragg formulated the diffraction law famously known as Bragg's law, who demonstrated the use of diffraction pattern to determine the crystal structure of $\mathrm{NaCl}^{7}$. His father, W. H. Bragg, had pioneered the design of instrumentation to observe diffraction patterns. Bragg's law (named only after the son, W. L. Bragg) was used to analyze the diffraction pattern and their joint and independent studies earned the Braggs (father and son) the Nobel Prize in Physics in 1915. The importance and applications of X-ray diffraction in several scientific fields have led to the award of 29 Nobel Prizes so far. The successful inception of crystallography into many scientific fields has been recognized by the United Nations, which declared 2014 as the International Year of Crystallography. ${ }^{8}$ It is, therefore, important to mention a few ground-breaking discoveries that furthered the significance of crystallography.

After the first crystal structure of $\mathrm{NaCl}$ was determined by X-ray diffraction, it was the diamond structure that established the role of crystallography in understanding material properties. The hardness of diamond was established as a repetitive tetrahedral arrangement of carbon atoms in the crystal lattice (Fig. 1). ${ }^{9}{ }^{10}$ Subsequent structural determinations included the structures of hexamethylenetetramine, quartz, and hexamethylbenzene. It was around late 1920s, structural studies on water by W. H. Barnes were instrumental in providing unambiguous insights into hydrogen bonding in water crystals. ${ }^{11}$ Since then, X-ray diffraction has been a reliable technique to evaluate the three-dimensional structure of many other materials including elements, binary compounds, and even proteins.

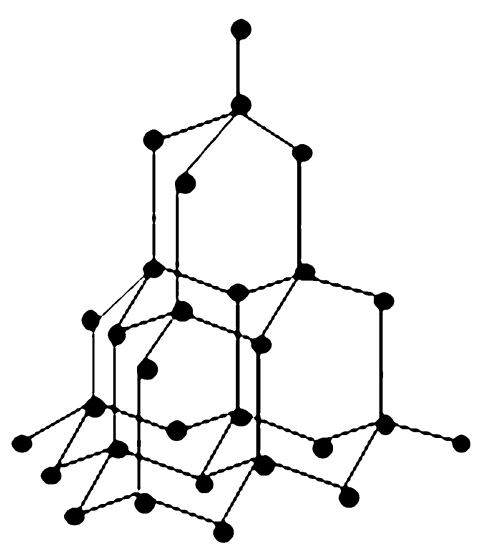

diamond

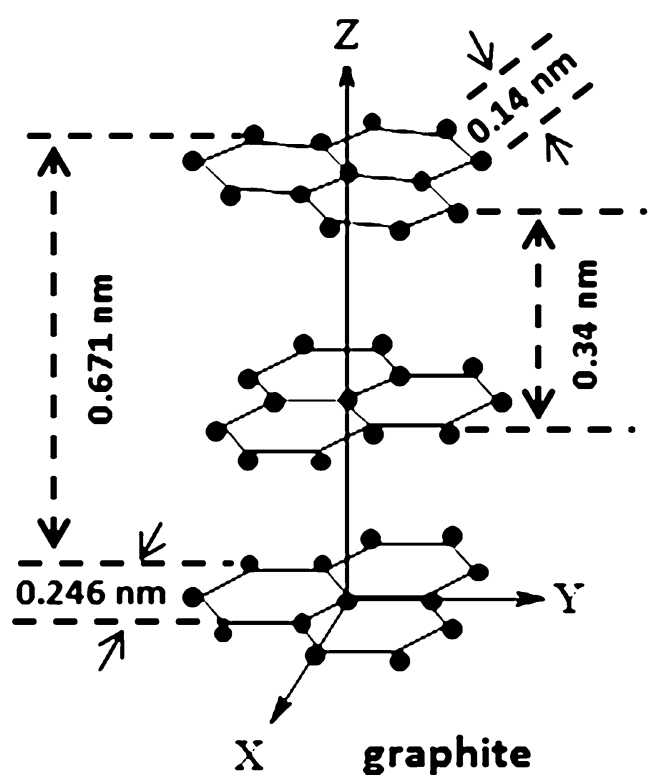

Figure 1: Diamond (left) and graphite (right), two allotropes of carbon, display distinct physical properties, which are rationalized on the basis of crystal structure analysis. Reproduced with permission from reference 10. Copyright @ 2006 The Royal Society of Chemistry. 
The first X-ray diffraction pictures from a small hydrated protein, pepsin, were collected by J. D. Bernal et al. in 1934. ${ }^{12}$ About 25 years later, crystal structures of myoglobin and hemoglobin were determined by J. Kendrew et al. in $1958^{13}$ and M. Perutz et al. in 1960, ${ }^{14}$ respectively, which earned them the Nobel Prize in Chemistry in 1962. D.C. Hodgkin solved the structures of a number of biological molecules, including cholesterol (1937), penicillin $(1949)^{15}$, vitamin B12 $(1954)^{16}$, and insulin (1969). ${ }^{8}$ Her seminal works were recognized with a Nobel Prize in Chemistry in 1964.

Chirality is fundamental in biology and drug molecules and has tremendous implications in drug-receptor interactions. The building blocks of proteins, the naturally occurring amino acids, are chiral. Milton and coworkers showed that inverting the chirality of an enzyme can lead to a complete inversion in its enantioselectivity. ${ }^{17}$ Thus, absolute configuration is critical for proper functioning of biological systems. Since these breakthroughs, the crystal structure of proteins, nucleic acids, and other biological molecules has been determined using X-ray crystallography. One of the biggest milestones of the 20th century was the discovery of the structure of DNA by J. D. Watson and F. H. C. Crick which was based on the diffraction experiments carried out by R. Franklin. ${ }^{18}$ Watson and Crick were awarded the Nobel Prize in Physiology in 1962, together with M. Wilkins, who had worked with Franklin. The discovery of the 'double helix' paved the way to macromolecule and protein crystallography, essential tools of the biological and medical sciences today.

Drug design strongly relies on the use of X-ray crystallography. A pharmaceutical company looking for a new drug to combat a specific bacterium or virus first needs to find a small molecule capable of blocking the active proteins (enzymes) that are involved in attacking the human cell. Knowing the precise shape of the protein allows scientists to design drug compounds that can clamp onto the 'active' site on the protein and thereby disable their harmful activity. With the advent of powerful X-ray sources (e.g., synchrotrons), new detectors, new experimental protocols, and the development of modern software, the number of accurately determined crystal structures of small and macromolecules has exponentially increased. Currently, the Cambridge Structural Database (CSD), ${ }^{19}$ which is the storehouse of small molecule crystal structures, contains more than 750,000 structures, and the Protein Data Bank ${ }^{20}$ over 105,000 structures of biomacromolecules, almost $90 \%$ of which were determined by means of X-ray diffraction.

\subsection{Modern developments: structure from powder diffraction}

Many crystalline solids exist as microcrystalline powders and making single crystals suitable for structure determination using single-crystal $\mathrm{X}$-ray diffraction technique is a daunting task. In these instances, X-ray powder diffraction provides an alternative tool for characterization of the crystalline powder materials. ${ }^{21}$ Over the past century, X-ray powder diffraction has evolved as one of the most potential characterization tools for qualitative and quantitative analysis of powder materials. The technique is non-destructive and has been applied to analyze phase purity of a wide range of materials from various disciplines such as geology, polymeric, environmental, forensic, and pharmaceutical sciences (Fig. 2). ${ }^{22}$ Seminal works of P. Debye and P. Scherrer on LiF in $1916^{23}$ and A. W. Hull on iron and various other materials in $1917^{24}$ mark the beginning of using $\mathrm{X}$-ray powder diffraction for material characterization. For over 50 years, it was primarily used for phase identification in mineralogy, metallurgy, and solid-state chemistry, and its extension to more complex problems, such as structure refinement and structure determination, was limited to only simple high symmetry systems. The pioneering contributions of $\mathrm{H}$. Rietveld heralded a paradigm shift by extending the reach of powder methods from simple high symmetry structures to complex low symmetry ones. There were a number of successful attempts to solve new structures from powder data by 1970s; however, these attempts were primarily based on trial-and-error methods. Since high-resolution powder diffractometers, such as synchrotron X-ray and pulsed neutron sources, became available, the field of structure determination has transformed from an art to science. The first challenge in structure determination is to index the diffraction pattern, but this was conveniently achieved with highresolution data. It was then possible to decompose the powder pattern into an approximate set of resolved, integrated intensities, so that the conventional structure-solving tools of the time could be applied. This met with a striking success in the mid-1980s. The unknown structure of iron (III) arsenate $\left(\mathrm{FeAsO}_{4}\right)$ was solved from high-resolution neutron data using direct methods, namely, the Patterson methods. ${ }^{25}$ So-called $\mathrm{ab}$ initio determination of structures from powder data has become routine in recent years.

Structure determination from powder X-ray diffraction data has potential applications in many important fields where obtaining single crystals for crystal structure determination is a
Chirality: It is a geometric property of some molecules and ions. A chiral molecule/ ion is non-superimposable on its mirror image (Greek: cheir $=$ hand). The presence of an asymmetric carbon centre is one of several structural features that induce chirality in organic and inorganic molecules.

Absolute configuration: Spatial arrangement of the atoms of a chiral molecular entity (or group) and its stereochemical description, e.g., $\mathrm{R}$ or $\mathrm{S}$, referring to Rectus, or Sinister, respectively. 


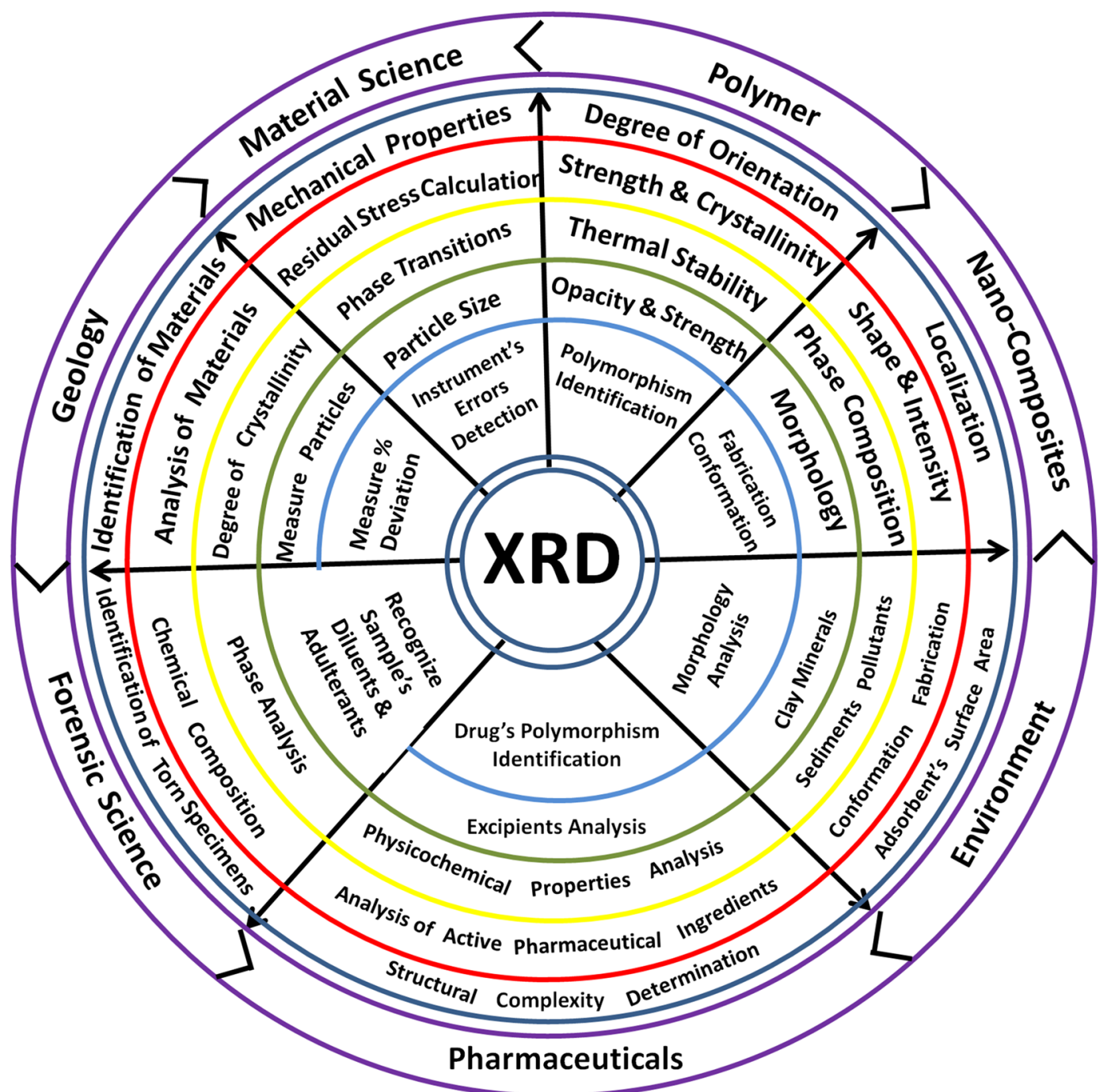

Figure 2: Analytical applications of powder X-ray diffraction in various fields. Reproduced with permission from reference 22. Copyright (c) 2014, Reviews on Advanced Materials Science.

challenging task. For example, the intermediates and products of solid-state reactions could be analyzed by powder diffraction for structural characterization without the need for crystallization. Multi-component crystals of the type of pharmaceutical cocrystals are often prepared by solid-state techniques and many cocrystals can be prepared only by grinding techniques. The microcrystalline powders thus obtained are generally not suitable for structural characterization by single-crystal X-ray diffraction. Therefore, powder X-ray diffraction is an alternative to investigate the structural features of cocrystals. For example, K. D. M. Harris and coworkers have demonstrated the use of powder X-ray diffraction to determine the crystal structure of a cocrystal involving racemic bis--naphthol, benzoquinone, and anthracene. ${ }^{26}$ Powder X-ray diffraction has also found numerous applications in pharmaceuticals. ${ }^{27}$ Active pharmaceuticals often go through a wide range of processes at preformulation and formulation stages of drug development. Hence, powder X-ray diffraction plays a key role in the evaluation of stability and possible phase conversions during manufacturing and formulation development. Many a times, solids obtained from these processes are not subjected to further crystallization and powder diffraction provides the only possible route for determining the crystal structure. Crystal structures of many pharmaceutical substances have been determined from powder diffraction data-to name a few, polymorphs of fanaserine, talmisartan, fluticasone propionate, and tetracaine hydrochloride. 


\subsection{Different Solid Forms and Their role in Drug Development}

Pharmaceuticals typically consist of one or more of APIs which are often formulated into a suitable final form comprising inactive ingredients. In the context of intrinsic value, APIs are the most valuable materials. A majority of drug products in the market are developed in the form of solids due to ease of making and stability advantages. Amongst the various critical issues in drug development, tackling poor physicochemical properties, such as solubility, stability dissolution rate, hygroscopicity, and permeability, remain of paramount importance. If a particular solid form has any issue(s) with physicochemical properties, drug developers often look for alternative solid forms, such as amorphous forms, polymorphs, salts, solvates/hydrates, and cocrystals. ${ }^{28}$

Crystalline solids have regular arrangement of molecules with repeating units in three dimensions, whereas amorphous solids lack long-range order (Fig. 3). Amorphous solids display desirable pharmaceutical properties, such as faster dissolution rate and bioavailability than its crystalline form, but they are not as much frequently marketed as crystalline solids owing to their poor physical stability and tendency for crystallization. ${ }^{29}$ Hence, a major fraction of marketed pharmaceuticals consists of APIs in crystalline form. These solids can show polymorphism, which is defined as the ability of a substance to exist in two or more crystalline phases that have different arrangements of molecules in crystal lattice.
Developing a polymorphic form is a promising route as it is the only alternative crystalline form in the pure form of drug substance. However, polymorphic variants only show a marginal solubility improvement and stability is a major concern as the metastable (unstable) polymorphs tend to convert to stable form impacting shelf-life and drug performance. Hydrates and solvates are crystalline solids that contain water and solvents, respectively, in the crystal lattice. Though some of the existing drug products are developed using hydrates or solvates, they are not often regarded as preferred solids owing to their low thermal stability, variable stoichiometry, and limited advantages with physicochemical properties. A pharmaceutical salt is formed when an acidic or basic drug reacts with basic/acidic salt former. The presence of ions in the crystal lattice strongly influences the physicochemical properties of the API, and the salt formation is the most preferred route in the development of APIs as drug products. However, a major setback with salt formation is that the technique is only applicable to the APIs, which are ionizable under normal $\mathrm{pH}$ conditions. Solid solutions and eutectic compositions, which are crystalline variable stoichiometry multi-components and conglomerates of solid solutions, respectively, are relatively less studied in pharmaceuticals but have also been explored for development of drug products. Over the past two decades, cocrystals offered a promising alternative route to gain control over physicochemical properties of APIs by judicious selection of

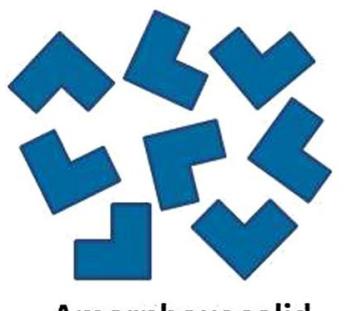

Amorphous solid

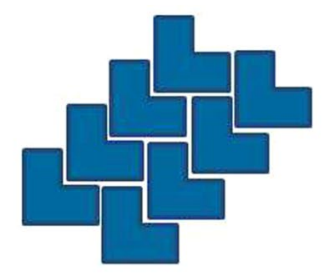

Polymorphs

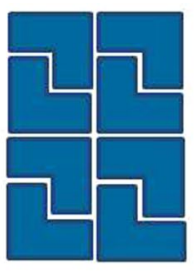

\section{.}

Salt

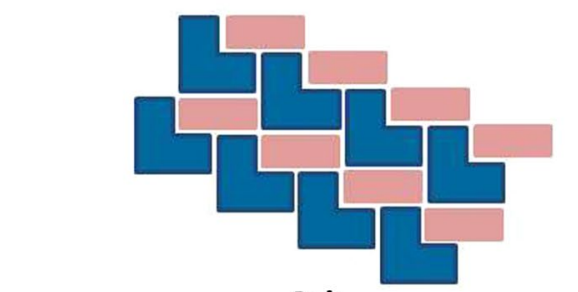

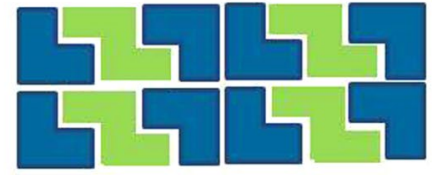

Cocrystal

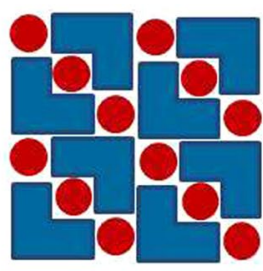

Hydrate/solvate

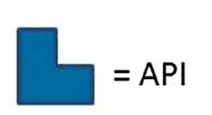

= counter ion
$=$ water $/$ solvent

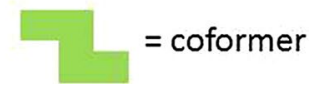

Bioavailability: It refers to the extent and rate at which the active moiety (drug or metabolite) enters systemic circulation, thereby accessing the site of action. Bioavailability of a drug is largely determined by the properties of the dosage form, which depend partly on its design and manufacture. Differences in bioavailability among formulations of a given drug can have clinical significance; thus, knowing whether drug formulations are equivalent is essential. 
coformers. ${ }^{30}$ The advantages with cocrystals over other traditional solid forms are discussed in the following sections.

\section{Cocrystals in Pharmaceutical Design 2.1 Modern Drug Development: Need for Better Solid Forms}

From finding a drug candidate to bringing a drug product to the market, a staggering amount of time is spent in its development. It has been estimated that an average of 12 years is required for total drug development and it costs about US $\$ 1.5$ billion. ${ }^{31}$ In the process, thousands of compounds are computationally screened and tested for their biological activity, and of the 500010,000 compounds that show promise, five will go to human trials, and only one will finally become an approved drug. Despite these hardships, if the API identified as suitable candidate does not offer promising physicochemical properties, such as solubility, stability, and dissolution rate, it significantly impacts drug development from an economic perspective. It is also to be highlighted that despite enormous computational and experimental advances, the number of new chemical entities that are used as drug substances has significantly declined. Therefore, modern drug development is not only focused on the discovery of new chemical entities, but also on exploring ways in which the existing drug substances can be used effectively. Such a repurposing of existing drug substances mainly refers to studying the drugs that are already approved to treat one disease or condition to see if they are safe and effective for treating other diseases. ${ }^{32}$ Alternatively, it could also mean improvement of physicochemical properties of active ingredients so as to develop better drugs with improved drug performance. This is often carried out by physical transformation of an API by making solid dispersions, micronization, and amorphous solid forms embedded within a polymer matrix. ${ }^{33}$ An alternative approach that has tremendous implication in drug development is to make novel solid forms of APIs, such as salts, polymorphs, solvates, hydrates, and cocrystals. Irrespective of the solid form chosen for development of a drug, APIs are seldom administered alone; rather they are given as part of formulation in combination with other inactive substances which aid the development of a suitable formulation for an appropriate mode of administration. While the biological action of the drug product depends on the activity of the API, its physicochemical properties determine how well the API is absorbed in the body to perform its action. Therefore, it is important to understand the physical description of the API prior to development of a dosage form. As discussed in the previous section, a given API can form a wide variety of solid forms and each has its own physicochemical properties, which could impact the formulation development. Thus, the complex nature of formulation development and manufacturing operations demands a closer look at how the properties of a given drug vary from one solid form to the other.

Pharmaceutical salts are by far the most commonly used solid forms for development of drug formulations. It is estimated that approximately $50 \%$ of drugs contain the active ingredient in salt form. ${ }^{34}$ Salt formation has a number of potential advantages. For example, salts are known to improve solubility, dissolution rate, melting point, photostability, processability, better taste, etc. A large number of monographs and books highlight the importance of salt formation in drug development. Despite widespread application of salt formation in drug development, the technique is limited to only APIs that are ionizable under normal $\mathrm{pH}$ conditions. Many drug products and drug substances in the developmental stages contain APIs that fall into this category. Therefore, alternative technique such as pharmaceutical cocrystallization is important for probing solid-form diversity of APIs. Cocrystallization scores additional advantages over salt formation as it can be applied even to non-ionizable APIs. Pharmaceutical cocrystals are made with coformers that are chosen from the list of Generally Recognized As Safe (GRAS) chemicals. GRAS chemicals are food additives considered safe for human consumption ${ }^{35}$ and their list constitutes a wide range of chemicals like aldehydes, alcohols, carboxylic acids, amides, and sweeteners. Therefore, the diversity of GRAS chemicals in terms of their structure and physicochemical properties provides an additional means of selecting a suitable coformer for a target change in the API. Over the past decade, alongside other well-known solid forms, such as salts, polymorphs, and hydrates/ solvates, cocrystals have shown immense promise in easing formulation development by addressing the physicochemical problems of APIs.

\subsection{Impact of cocrystals on drug development}

Physicochemical properties of an API differ from one solid form to the other which is often a consequence of the way the API molecules are arranged in the crystal lattice of that solid form. 
Hence, selection of a solid form has profound implications in clinical, legal, and regulatory perspective. It has been estimated that approximately $80 \%$ of the drugs currently under development belong to Class II or IV of the biopharmaceutical classification system (BCS). Poor solubility is the characteristic nature of the drugs that fall into these classes. Drug formulators often rely on techniques such as micronization, solid dispersion, encapsulation, salt formation, amorphous forms, etc., for addressing solubility issues. However, these techniques have inherent drawbacks in manufacturing and additional risk of stability of resulting formulations. Cocrystals (Table 1) are attractive solid forms for addressing solubility issues of BCS Class II and IV drugs. In addition, many solid-state problems such as physical stability, hygroscopicity, melting point, and dissolution rate could be modified by selecting an appropriate coformer. ${ }^{28,}{ }^{30}$ From an intellectual property perspective, the emergence of cocrystals as novel solid forms provided new avenues for extending patent life of parent drugs. Cocrystals that show improved physicochemical properties satisfy the three criteria required for issuing a patent: (1) novelty, every cocrystal is novel as it is not possible to predict whether a combination of an API and coformer forms a cocrystal, (2) non-obviousness, the physicochemical and pharmacokinetic properties of APIs are difficult to predict unless detailed experiments were conducted, (3) utility, the main motivation in the development of cocrystals is due to their ability to improve the performance of the parent drug. A. $\mathrm{V}$. Trask puts forth the patentability aspects of pharmaceutical cocrystals. ${ }^{36}$ With the interest in pharmaceutical cocrystals growing, there is a plethora of patent applications on cocrystals. The potential applications of cocrystals in the development of drug formulations has been recognized by several pharmaceutical companies and many have even recognized the need for their screening as part of solid-form screening for identifying an optimal solid form for development. The importance of pharmaceutical cocrystals has been recognized recently by the US Food and Drug Administration (FDA, 2011) ${ }^{37}$ and European Medicines Agency (EMA, 2014), ${ }^{38}$ which released draft guidelines on the subject of regulatory classification of pharmaceutical cocrystals. While FDA classified pharmaceutical cocrystals as drug product intermediates and the coformers used to make the cocrystals are defined as excipients, EMA defined them as being solidstate variants of the APIs, aligning them with salts, polymorphs, hydrates, or solvates. The views
Encapsulation: It is a process in which particles of a substance are surrounded by a coating to give capsules of many useful properties. In pharmaceuticals, encapsulation of drug molecules into microspheres or nanoparticles enables controlled drug release, taste masking, improving stability, and protection of protein and peptide from degradation.
Table 1 Milestones in the area of cocrystal research

\begin{tabular}{|c|c|}
\hline Year & Milestone \\
\hline 1844 & $\begin{array}{l}\text { Discovery of the first cocrystal by F. Wöhler involving benzoquinone and hydroquinone (termed as quinhy- } \\
\text { drone) }\end{array}$ \\
\hline 1963 & $\begin{array}{l}\text { First use of the term "cocrystal" by W.R. Lawton and E.F. Lopez for crystalline complexes involving organic } \\
\text { amines and bisphenol }\end{array}$ \\
\hline $\begin{array}{l}1974- \\
91\end{array}$ & $\begin{array}{l}\text { Several drug-drug molecular complexes (cocrystals) involving antipyrene and salicylic acid, theophylline and } \\
\text { phenobarbital, pyrimidine and barbituric acid, and sulfaproxyline and caffeine, etc }\end{array}$ \\
\hline 1990 & $\begin{array}{l}\text { "Rules" of hydrogen bonding proposed by M.C. Etter are useful for predicting hydrogen-bond patterns in } \\
\text { organic crystals (including cocrystals) }\end{array}$ \\
\hline 1995 & $\begin{array}{l}\text { Introduction by G.R. Desiraju of the concept of supramolecular synthons in crystal engineering. This has had } \\
\text { profound impact on the design of cocrystals }\end{array}$ \\
\hline 2004 & $\begin{array}{l}\text { Seminal feature article by Ö. Almarsson and M.J. Zaworotko in Chemical Communications: Formal begin- } \\
\text { ning of the subject of pharmaceutical cocrystals }\end{array}$ \\
\hline 2005 & $\begin{array}{l}\text { An article by M.J. Zaworotko and coworkers that raised, for the first time, the question: are cocrystals less or } \\
\text { more prone to polymorphism? }\end{array}$ \\
\hline 2007 & A comprehensive review by A. V. Trask on patentability aspects of pharmaceutical cocrystals \\
\hline 2009 & Indo-US Workshop on pharmaceutical cocrystals and polymorphs \\
\hline 2011 & US-FDA's guidance on regulatory classification of cocrystals \\
\hline 2014 & EMA's reflection paper on the use of cocrystals in medicinal products \\
\hline 2015 & $\begin{array}{l}\text { Entresto: first marketed cocrystal post US-FDA's guidance (2011) on cocrystals that consist of disodium val- } \\
\text { sartan and monosodium sacubitril. The drug was launched by Novartis for the treatment of chronic heart } \\
\text { failure }\end{array}$ \\
\hline 2016 & US-FDA's revised guidance on cocrystals, which eases the regulatory burden of pharmaceutical cocrystals \\
\hline
\end{tabular}


of the regulatory bodies, in particular FDA, are contrary to what the current understanding of the concept of pharmaceutical cocrystals is that the second component in a cocrystal is a pharmaceutically acceptable coformer. In addition, by classifying them as drug product intermediates, cocrystals have to comply with additional current good-manufacturing practice requirements (cGMPs). Therefore, to address the industry concerns and also to ease the regulatory burden, the FDA has most recently reclassified them (August 2016) as a special class of solvates in which the second component is non-volatile. ${ }^{39}$ Furthermore, the FDA made sure that their regulatory classification is similar to that of a polymorph of the API, meaning that cocrystals do not require regulatory approvals as is required by a new API or a drug intermediate.

\subsection{Molecular Insights into Cocrystal Formation}

Hydrogen bonds: It is an attractive interaction between

a hydrogen atom from a molecule or a molecular fragment $\mathrm{X}-\mathrm{H}$ in which $\mathrm{X}$ is more electronegative than $\mathrm{H}$, and an atom or a group of atoms in the same or a different molecule, in which there is evidence of bond formation.

Hydrogen-bonding rules: (1) All good proton donors and acceptors are used in hydrogen bonding. (2) Sixmembered-ring intramolecular hydrogen bonds form in preference to intermolecular hydrogen bonds. (3) The best proton donors and acceptors remaining after intramolecular hydrogen-bond formation

will form intermolecular hydrogen bonds to one another. These rules correlate functional groups in neutral organic molecules with

hydrogen-bond patterns in crystals and useful for selection of suitable coformers for the design of cocrystals.

Supramolecular synthons: Structural units within supermolecules (crystals) that can be formed and/or assembled

by known or conceivable synthetic operations involving intermolecular interactions. Design of cocrystals generally relies on identification of robust supramolecular synthons between the constituents of the cocrystal.
Cocrystals are defined as multi-component crystals composed of two or more solid components in stoichiometric ratio. ${ }^{40}$ Non-covalent interactions of the type hydrogen bonds are often responsible for the association of solid components in a cocrystal. Therefore, knowledge of intermolecular interactions between the molecular components is a prerequisite for a successful cocrystal design. In this regard, G.R. Desiraju's supramolecular synthon concept $t^{41}$ and M.C. Etter's hydrogen-bonding "rules" 42 provide valuable insights into the association of two or more molecules in the crystal lattice. Recurring intermolecular interactions in a crystal structure are termed supramolecular synthons and are further classified into two basic types. One that involves the same functional groups is called supramolecular homosynthon and the one that involves different but complementary functional groups is called supramolecular heterosynthon. As the cocrystals are formed due to intermolecular interactions between two or more different molecules, the supramolecular heterosynthons of the type that involve functional groups of different molecules play an important role in the design of cocrystals.

Crystal engineering was defined by G. R. Desiraju as "the understanding of intermolecular interactions in the context of crystal packing and in the utilization of such understanding in the design of new solids with desired physical and chemical properties" reinforces the molecular recognition between molecules through a knowledge of intermolecular interactions. ${ }^{43}$ Therefore, crystal engineering strategies serve as guiding principles for the design of cocrystals. The knowledge obtained from the analysis of the crystal structures deposited in the Cambridge Structural Database (CSD) is used in the selection of coformers for cocrystal formation. A successful cocrystal design demands supramolecular synthons that are observed most frequently between the functional groups present on the components of cocrystals. Hence, the design of cocrystals for a given molecule starts with analyzing the functional groups available on that molecule and finding complementary functional groups which would likely form predictable supramolecular synthons. Thus, the coformer selection in a cocrystal design strategy reinforces a greater role of the knowledge of intermolecular interactions which is often drawn from X-ray crystal structure analysis.

\section{Physicochemical Properties of Pharmaceutical Cocrystals}

Cocrystallization is the result of molecular recognition between two or more different molecules through energetically favorable intermolecular interactions. The strength of intermolecular interactions and the way that the constituents of the cocrystal arranged in the crystal lattice determine its physicochemical properties. Hence, it is important that a detailed structural analysis is a prerequisite for understanding the physicochemical properties, which not only results in structure-property correlation, but also aids in subsequent design of cocrystals for fine-tuning the API properties. Over the past century, single-crystal X-ray diffraction has proven to be an important tool for unambiguous determination of crystal structures, and thus, assisted in ground-breaking analysis of material properties. With respect to cocrystals, structural characterization (a) establishes the reliability of cocrystal design strategy, (b) reveals hydrogenbond preferences of the functional groups, and (c) provides insights into structure-property correlation.

The existing vast literature on cocrystals provides a breadth of knowledge on their diverse applications. These primarily deal with improvement in solubility, dissolution rate, stability, color, melting point, mechanical strength, etc. ${ }^{28,} 30$ Recently, cocrystals have also been found to modify properties such as permeability of drugs, ${ }^{44}$ explosive nature of energetic materials, ${ }^{45}$ non-linear optical properties of organic crystals. ${ }^{46}$ This section of the review is exclusively focused on 
some properties of cocrystals for which X-ray diffraction is instrumental in evaluating the structure-property correlations.

\subsection{Reduced Solubility of Sulfacetamide for Improved Drug Residence Time}

Solubility is an important parameter in determining the bioavailability of a drug. Pharmaceutical cocrystals have proven to be valuable for addressing solubility and dissolution rate issues of several BCS Class II and IV drug substances, for which low solubility is a serious concern in development of drug formulations. While a large number of cocrystals have been reported that showed improved solubility of API, attempts to reduce the solubility of an API through cocrystallization are seldom reported. One such study that has demonstrated the application of cocrystallization technique in controlling the solubility of an API was reported by A. Nangia and coworkers using an antibiotic, sulfacetamide (SACT). ${ }^{47}$ SACT is a commonly prescribed topical antibiotic to treat conjunctivitis and other ocular ailments; however, its therapeutic efficacy is severely limited by physiological constraints such as tear flow and reflex blinking, and results in considerable drug loss. Such a limitation necessitates frequent dosing, which is often inconvenient to patients and amounts to excess drug loading. The authors have hypothesized that if the weaker hydrogen-bonding synthons in the crystal structure of SACT are replaced with stronger hydrogen bonds by way of cocrystallization, then the resulting stable cocrystal may be useful to lower the solubility of the parent drug. Crystal structure of the parent SACT features self-assembly of the SACT molecules through intermolecular $\mathrm{N}-\mathrm{H}$... O hydrogen bonds supported by a single auxiliary $\mathrm{C}-\mathrm{H}$-.. O interaction. By employing a crystal engineering strategy, the authors have prepared a series of cocrystals and analyzed crystal structures and evaluated the physicochemical properties. Interestingly, the cocrystals showed low solubility and dissolution rate compared to the parent API. The crystal structure of sulfacetamide-caffeine (SACT-CAF) cocrystal features the SACT molecules propagating through stronger heteromeric interactions of the type $\mathrm{N}-\mathrm{H} \cdots \mathrm{O}$ and $\mathrm{N}-\mathrm{H} \cdots \mathrm{N}$ hydrogen bonds and further stabilized by $\mathrm{C}-\mathrm{H} \cdots \mathrm{O}$ interactions (Fig. 4). The heteromeric interactions afford the cocrystal better packing efficiency and stronger crystal lattice, which showed lower solubility and dissolution rate of SACT. It has also been found that the cocrystal is stable under slurry and accelerated test conditions - a consequence of the stronger crystal lattice of the cocrystal. The low solubility and good stability of the SACT-CAF
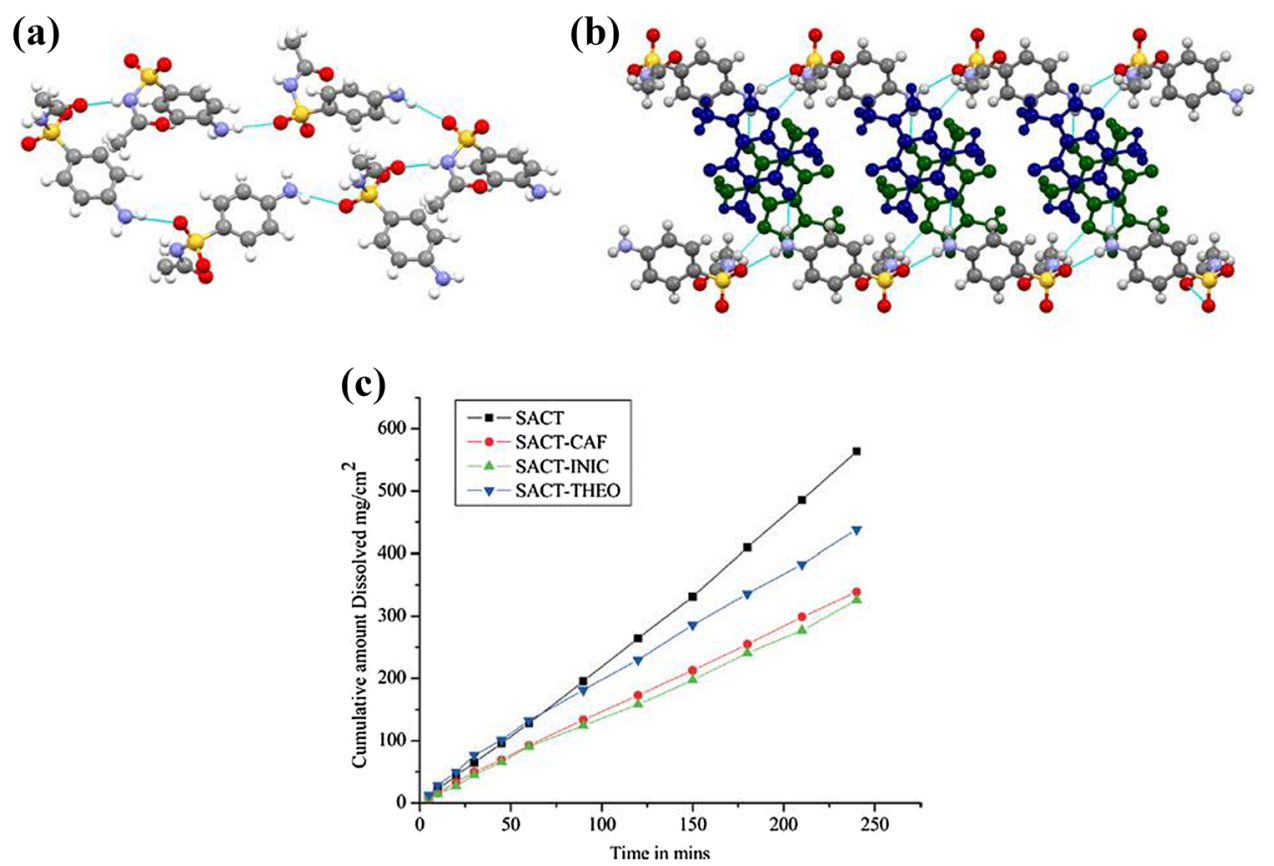

Figure 4: Crystal packing in the parent SACT (a), SACT-CAF cocrystal (b), and comparison of the dissolution rate of cocrystals and the API (c). Reproduced with permission from reference 47 . Copyright $($ ) 2014, The Royal Society of Chemistry. 
cocrystal could potentially address the poor residence time and faster elimination issues of SACT similar to extended release formulations.

\subsection{Improved permeability of hydrochlorothiazide through cocrystallization}

Permeability across barrier tissue, for instance, gastrointestinal mucosa, determines absorption and distribution of drugs. To improve permeability, lipophilic and non-ionizable drugs are preferred, but such drugs can cause poor solubility and thus result in low oral absorption. Permeability along with solubility may provide an insightful understanding of bioavailability. The amount of drug transported at the membrane is a function of the concentration gradient. Hence, high-soluble drugs may be absorbed well. The diffusion behavior is also dependent on the particle size. The in vitro diffusion measurements are commonly performed using parallel artificial membrane permeability assay and Caco- 2 cells.

Cocrystallization of APIs for improving aqueous solubility has been a well-established technique; however, studies on improving drug permeability through cocrystallization have been reported only recently. G. R. Desiraju and coworkers demonstrated applications of cocrystals in improving the permeability of a BCS Class IV, diuretic drug, hydrochlorothiazide (HCT) ${ }^{48}$ Five cocrystals with piperazine (PPZ), picolinamide (PCM), tetramethylpyrazine (TMPZ), malonamide (MAM), or isoniazid (INZ) were prepared and their solubility and permeability evaluated using a Franz diffusion cell. The solubility and permeability product best represents the impact of cocrystallization on pharmacokinetic properties of HCT. It has been found that the cocrystals HCT-PPZ and HCT-PCM showed large enhancement in solubility and flux/permeability compared to HCT itself (Fig. 5). Notably, HCT-PPZ cocrystal shows the highest solubility and permeability among all the known HCT cocrystals. The diffusion behavior of HCT-MAM, HCT-INZ, and HCT-TMPZ was marginally higher than that of HCT. The observed improvement in solubility and permeability of the cocrystals is due to the disruption of sulfonamide-sulfonamide homosynthon in API by the coformer molecules. For example, crystal packing of the HCT-PPZ cocrystal features a 2D bilayer of HCT molecules via $\mathrm{N}-\mathrm{H} \cdots \mathrm{O}$ and $\mathrm{C}-\mathrm{Cl} \cdots \mathrm{O}=\mathrm{S}$ interactions, and these layers bridged through $\mathrm{PPZ}$ molecules via $\mathrm{N}-\mathrm{H} \cdots \mathrm{N}$ hydrogen bonds (Fig. 6). The authors proposed that the higher concentration of cocrystals in solution leads to amorphous cocrystal formation in solution with moderate or weak hydrogen bonds, which could generate high concentration gradient at the membrane site. Thus, the resulting high transient concentrations overcome lipophilicity and particle size effects and provide improved permeation.

\subsection{Improved Bioavailability of the Muscle Relaxant, Metaxalone}

Metaxalone (MTX) is a muscle relaxant used to relieve discomfort associated with acute and painful musculoskeletal conditions. Its effects have (a)

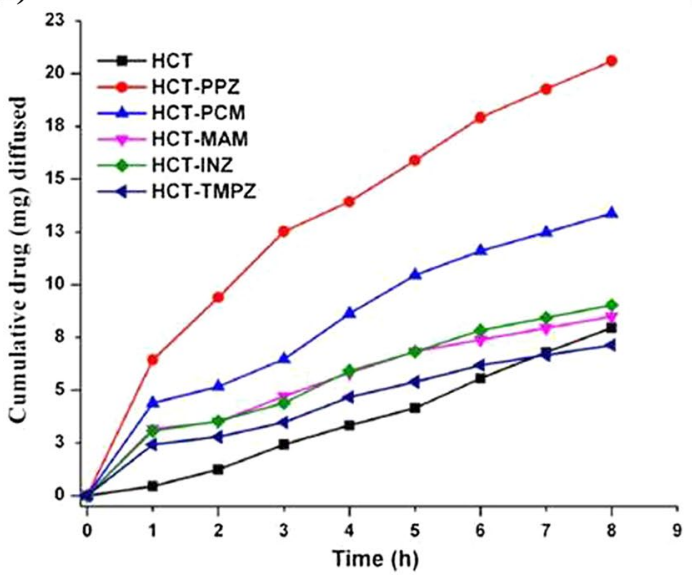

(b)

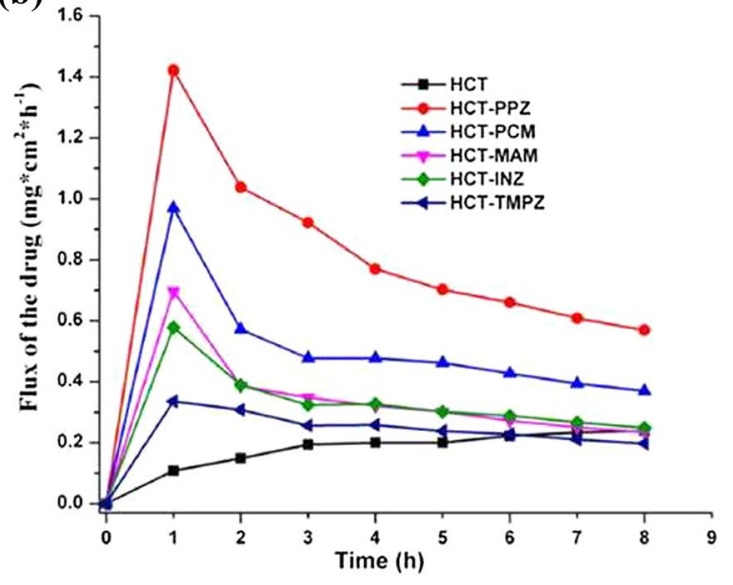

Figure 5: Results of permeability studies on HCT and its cocrystals, a cumulative amounts of cocrystals diffused vs time. b Flux/permeability of cocrystals vs time. Reproduced with permission from reference 48. Copyright @ 2016, The American Chemical Society. 
been reasoned to depression of central nervous system. ${ }^{49}$ MTX is currently available in market as Skelaxin ${ }^{\circledR}$ in tablets of strengths 400 and $800 \mathrm{mg}$. It belongs to Class II drugs of BCS and shows poor solubility. It has also been reported that the oral bioavailability of MTX is greatly influenced by food, which impacts dosing needs of a patient. The previous attempts to make a sufficiently bioavailable form of MTX have not been successful. MTX exists in two polymorphic forms. ${ }^{50}$ Crystal structures of the polymorphs feature distinct hydrogen bonding-one polymorph showing imide group of the oxazolidone ring to form imide-imide catemer synthon and the other to form imide-imide dimer synthon (Fig. 7). Nuformix Limited (UK) has recently pursued cocrystallization route to modify bioavailability of the MTX. Oxazolidone group of the MTX was exploited to form a series of cocrystals with dicarboxylic acids such as, succinic acid, fumaric acid, maleic acid, adipic acid, and salicylic acid. ${ }^{51}$ Shown in Fig. 7, the imide-imide dimer synthon persists in all the cocrystals, which are further hydrogen bonded with the coformers via strong hydrogen bonds. The presence of coformer in the crystal lattice resulted in a significant change in the bioavailability of the MTX. Pharmacokinetic (PK) studies in beagle dogs using cocrystals with succinic and fumaric acids revealed higher plasma concentration and the area under the curve (AUC) for cocrystals than the MTX. The promising PK properties make the MTX cocrystals valuable for development of novel MTX formulations and are currently in clinical trials. (a)

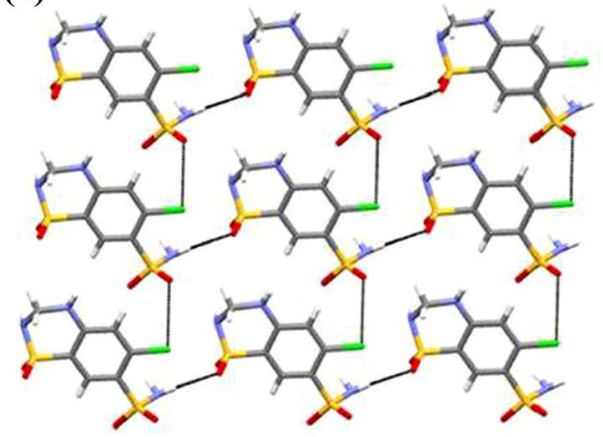

(b)

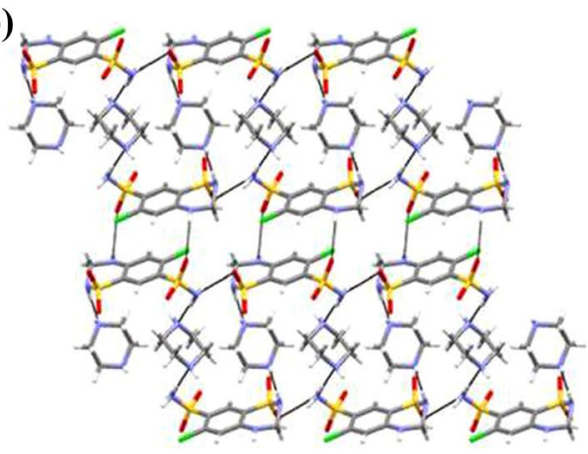

Figure 6: Packing of the HCT-PPZ cocrystal: a 2D HCT layers propagated along the ab-plane. b Packing of HCT bilayers through PPZ bridging. Reproduced with permission from reference 48. Copyright @ 2016, The American Chemical Society.

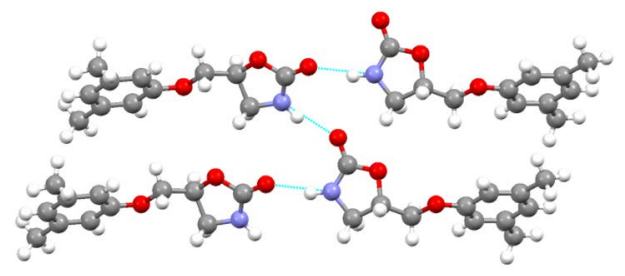

MTX Form A

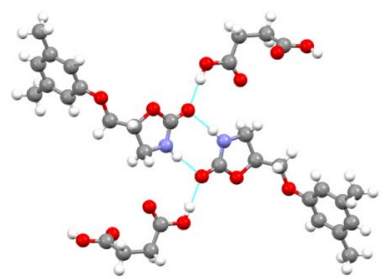

MTX-succinic acid cocrystal

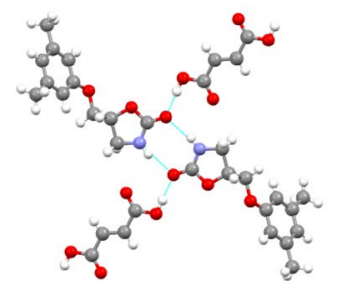

MTX-fumaric acid cocrystal

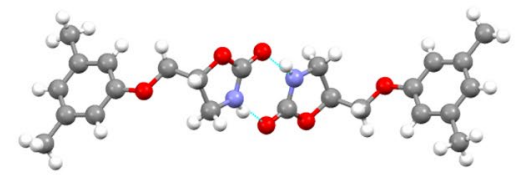

MTX Form B

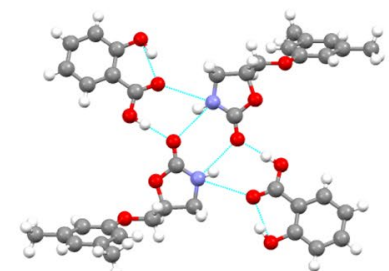

MTX-salicylic acid cocrystal

Figure 7: Hydrogen bonding in MTX polymorphs and its cocrystals. Notice that the imide-imide dimer synthon is retained in all the crystal structures of the cocrystals. Reproduced with permission from reference 50. Copyright @ 2011, The American Chemical Society. 


\subsection{Improved Photostability of Nitrofurantoin Through Cocrystallization}

The exposure of photons on pharmaceutical materials can have destructive effects impacting quality and efficacy of drug products. As per International Conference on Harmonisation $(\mathrm{ICH})$ guidelines, photostability testing is an integral part of pharmaceutical development process. The drug products undergo a variety of complex photochemical reactions depending upon the chemical and physical structure of the materials, such that the labile functionalities, such as conjugated double bonds or aromatic residues containing $\mathrm{N}, \mathrm{S}$, or $\mathrm{O}$, are prone to photochemical degradation.

Applications of cocrystals in modulating photochemical reactivity of pharmaceuticals have been paid less attention until recently. For example, V.R. Vangala and coworkers demonstrated for the first time that the improvement in photostability of an antibiotic drug, nitrofurantoin (NF), can be achieved via cocrystallization. ${ }^{52,53}$ Stability studies on NF powder sample revealed that upon irradiation at $315-400 \mathrm{~nm}$, $\sim 28 \%$ of the sample underwent degradation in a week (Fig. 8). Mechanistically, NF photodegradation was attributed to rapid isomerization at $\mathrm{C}=\mathrm{N}$ bonds and subsequent degradation to 5-nitro-2-furaldehyde and 1-aminohydantoin (Fig. 8a). By following a crystal engineering strategy, the authors have made a series of cocrystals and studied their photostability. As shown in Fig. 8b, the cocrystals with coformers, 4-hydroxybenzamide (4HBAM), 3-aminobenzoic acid (3ABA), 4-aminobenzoic acid (4ABA), and urea, all degraded to a minor extent $(<3 \%)$, except the cocrystal with urea, which showed only some degree of stabilization ( $\sim 17 \%$ degraded). Interestingly, under similar experimental conditions, photostability of physical mixtures of $\mathrm{NF}$ and coformers was similar to NF (26-30\%

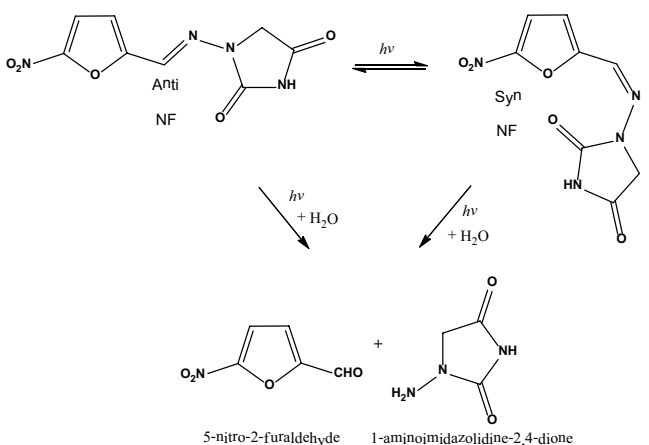

(a)

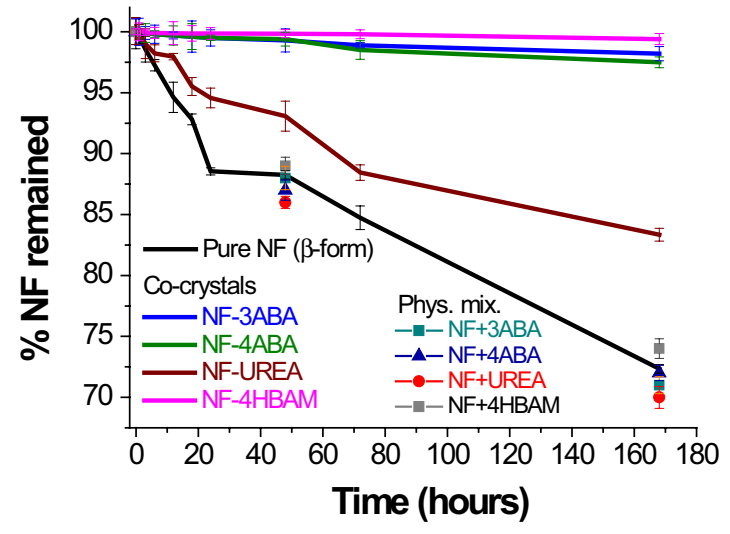

(b)

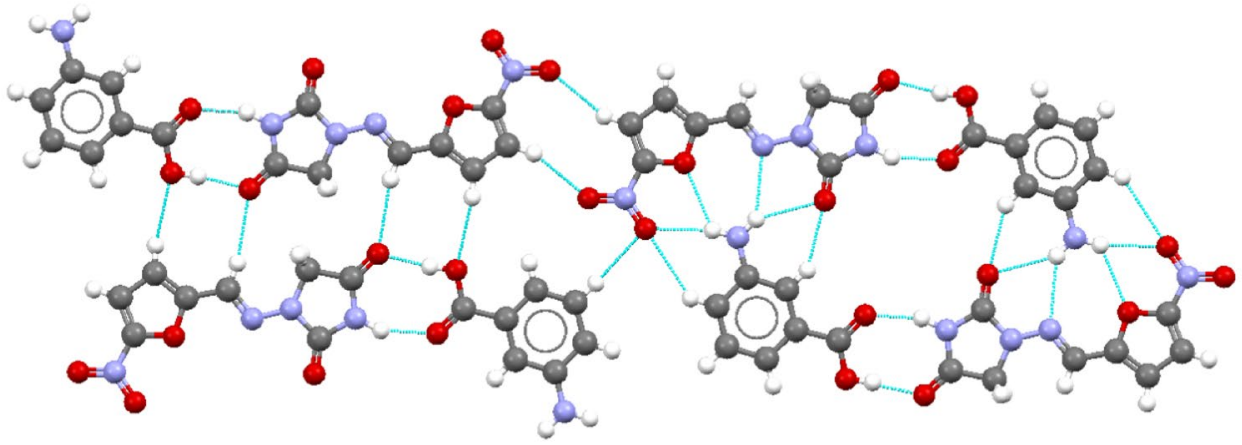

(c)

Figure 8: a Chemical degradation of nitrofurantoin. Note NF cleavage at azomethine ring, b UV irradiation of pure NF, cocrystals, and NF + coformer physical mixture samples up to a week and the photodegradation curves show the enhanced photostability of cocrystals, and c solid-state packing of NF-3ABA (1:1) and note the acid-imide heterosynthon between NF and 3ABA molecules. Reproduced with permission from reference 53. Copyright (c) 2012, The American Chemical Society. 
degradation), suggesting the potential of cocrystallization as a means to control the photodegradation of NF.

The greater photostability of the cocrystals was rationalized on the basis of crystal structures. For example, NF and 3ABA form planar hydrogenbonded sheets in the crystal structure of NF-3ABA cocrystal (Fig. 8c). A closer look into the crystal structure revealed some important structural features: (1) the acid group of 3ABA forms an acidimide heterosynthon (via $\mathrm{O}-\mathrm{H} \cdots \mathrm{O}$ and $\mathrm{N}-\mathrm{H} \cdots \mathrm{O}$ interactions) with NF, and (2) the amine group of 3ABA forms four strong hydrogen bonds with the remaining hydrogen-bond acceptors of the NF. The formation of multiple strong hydrogen bonds between the coformer and NF could potentially hinder the photoisomerization of $\mathrm{C}=\mathrm{N}$ and hence offer better photostability for the cocrystal. Similarly, greater photostability of the other cocrystals could be reasoned to the presence of supramolecular synthons between NF and coformers and stronger crystal lattice that hamper the photoisomerization of $\mathrm{NF}$ at the $\mathrm{C}=\mathrm{N}$ bond.

\subsection{Mechanical Property-Crystal Structure Correlations in Cocrystals of Paracetamol}

Materials undergo deformation on applying stress; this includes elasticity, plasticity, viscoelasticity, brittle fracture, fragmentation, or a combination of these based on the nature of applied stress and internal structure of the material. A proper understanding of these mechanical properties and material deformation phenomena is important for powder compaction and secondary processes such as milling. The previous studies on single-component systems are aimed at correlating compaction properties and plasticity of materials. The presence of slip planes and crystallographic planes with weakest interactions or adjacent planes with higher d-spacing in the crystal structure affords greater plasticity and
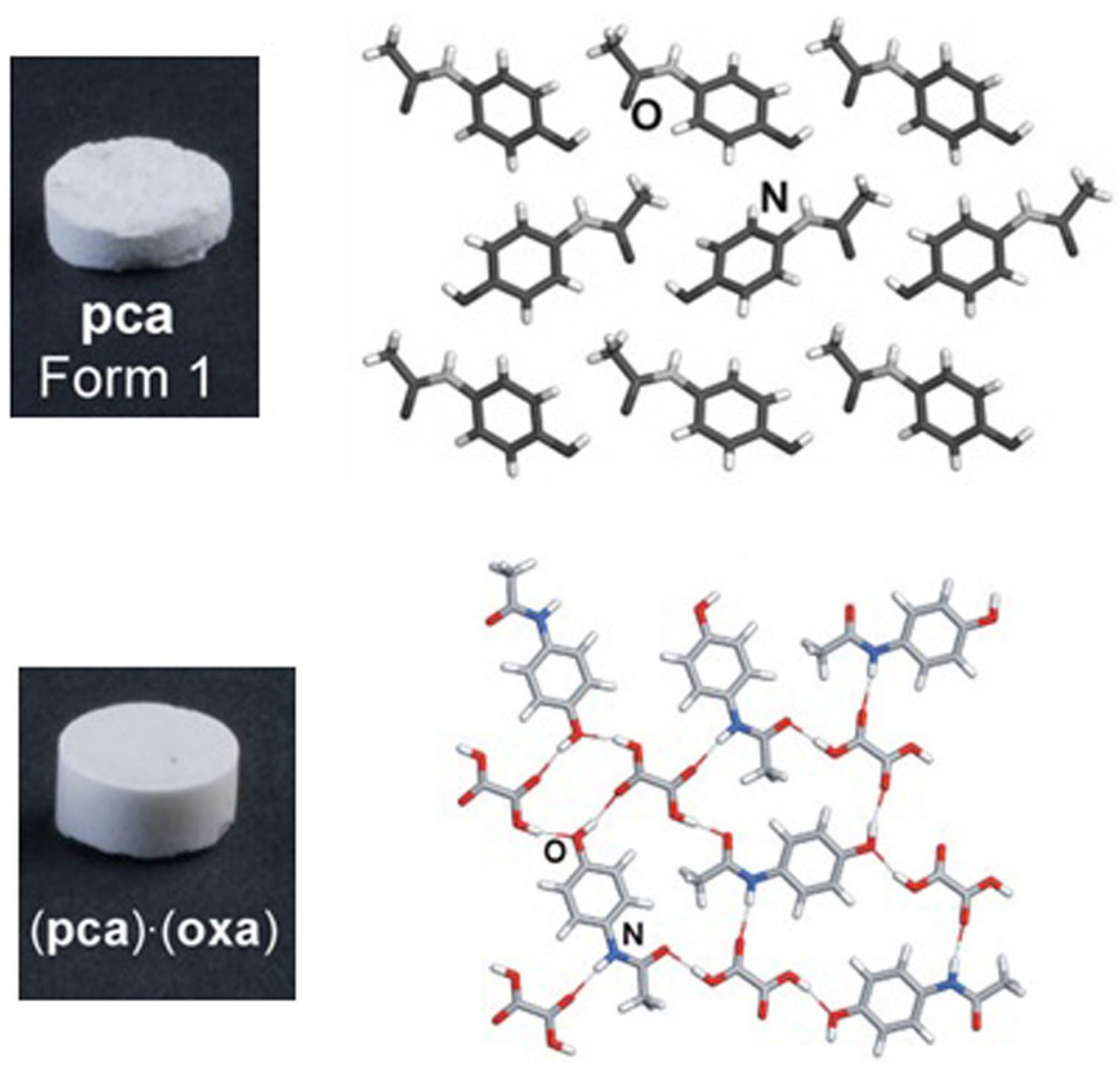

Figure 9: Comparison of compressed tablets and crystal structures of PRA and PRA-oxalic acid cocrystal. Notice that the perfect layered structure of the cocrystal affords improved tabletability. Reprinted with permission from reference 54. Copyright (C) 2009 by John Wiley and Sons, Inc. 
better tabletability. Studies concerning mechanical properties of cocrystals have been conducted only recently. For example, W. Jones and coworkers have found that the cocrystals of paracetamol (PRA) showed better tabletability than the marketed polymorph. ${ }^{54}$ Most recently, S. P. Velaga et al. have demonstrated the relationship between mechanical properties and crystal structure in PRA (form I) and its cocrystals. ${ }^{55}$ Thermodynamically stable Form I of PRA showed poor tabletability due to high elastic deformation (Fig. 9). However, cocrystals with oxalic acid and 4,4'-bipyridine, and a salt with $\mathrm{HCl}$ displayed superior tabletability compared to PRA. The tabletability of the substances is shown in the order of: OXA $>$ PRA-HCl $\approx$ PRA-OXA $>$ BPY $>$ PRABPY. A closer look into the crystal structures of the cocrystals and Form I revealed that the crystal structure of Form I has no slip plane and consisted primarily of interlocked PRA molecules held strongly by hydrogen bonds. The rigid hydrogen-bonded network affords poor tableting properties that resulted in brittleness to the compressed tablets. On the other hand, the cocrystal with oxalic acid features catemeric arrangement of oxalic acid and PRA molecules that generate layers parallel to (200) and (100) planes. The perfect layered structure of the cocrystal explains the greater plastic deformation and better tabletability. These studies underscore the role of crystal packing and the strength of intermolecular bonding in determining tablet formation and its mechanical strength.

\subsection{Marketability of Cocrystals}

Marketability of a solid form depends on several factors and its promising physicochemical properties are undoubtedly the prime consideration. As detailed in Sects. 3.1-3.5, cocrystals have proved successful in modifying physicochemical properties of several important APIs. The adoptability of cocrystallization technology to a wide range of APIs, including the ones that are nonionizable, has tremendous implications in drug development. The inherent characteristics of cocrystals such as novelty, usefulness, and nonobviousness provide additional means for patenting cocrystals as novel composition of matter. A close inspection of the marketed drugs and their composition revealed that some of them contain the API in cocrystal form. For example, well-known drugs such as caffeine citrate, sodium valproate-valproic acid, and escitalopram oxalate-oxalic acid, all comply with the cocrystal definition and marketed even before the concept of pharmaceutical cocrystals came to existence. ${ }^{40}$ The recognition by US-FDA and EMA has furthered the cocrystallization research into a main stream preformulation technique and validated the use of cocrystals for drug development. Most recently, US-FDA and EMA approved a cocrystal hydrate that contains disodium valsartan and monosodium sacubitril (Fig. 10). ${ }^{56}$ The cocrystal was developed by Novartis with the trade name Entresto and is used in the treatment of chronic heart failure. Some other promising cocrystals that are under late- stage development are: a drug-drug cocrystal containing a non-steroidal anti-inflammatory drug, celcoxib, and an opiod drug, tramadol, which has superior analgesic efficacy compared to comparable doses of tramadol, ${ }^{57}$ and a cocrystal of an anti-diabetic drug ertugliflozin with 5-oxo-proline. ${ }^{58}$

\section{Conclusions}

Since its discovery, X-ray crystallography remains at the forefront of structural characterization of materials. Recent developments such as evolution of synchrotron radiation and structure determination from X-ray powder diffraction data have

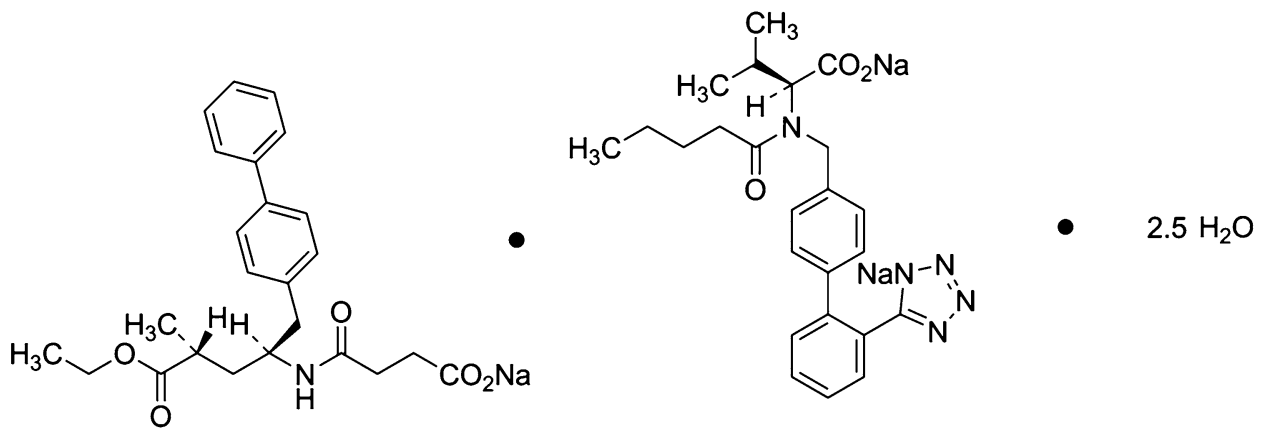

Figure 10: Molecular components of Entresto: disodium valsartan, monosodium sacubitril, and water which has been approved by FDA and EMA recently. 
furthered the applications of X-ray crystallography to characterization of a broader range of materials. The current ease of structure determination using X-ray crystallography has resulted in a paradigm shift from simple three-dimensional structural analysis to correlating material properties with the crystal structure. A wide range of material properties have successfully been rationalized on the basis of crystal structure, which not only unraveled many scientific mysteries but also aided rational design of functional materials. In pharmaceuticals, X-ray crystallography played a phenomenal role in understanding the properties of drug molecules, which has several important legal and economic impacts. The emergence of pharmaceutical cocrystals as alternatives to longknown salts, solvates/hydrates, polymorphs, etc., has provided additional means of fine-tuning physicochemical properties of drug molecules. In this regard, structural information-deduced from X-ray crystal structure analysis provides breadth of information for judicious selection of coformers for a target change in drug property. Changes in drug properties such as solubility, dissolution rate, physical and chemical stability, permeability, and hygroscopicity have successfully been correlated with the crystal structures of API and its cocrystals. It has been rationalized that the coformers that result in significant difference in the crystal structure of API have profound impact on drug properties. Therefore, design of pharmaceutical cocrystals demands knowledge of competition among various possible intermolecular interactions between API and coformers. Despite a significant development in the field of cocrystal design, there remain further challenges. For example, (1) the presence of multiple hydrogen-bonding sites on the API or the selected coformer increases the complexity and hinders application of the traditional cocrystal design strategies. Greater understanding of hydrogenbonding preferences of a particular functional group in the presence of other functional groups provides valuable insights into successful cocrystal design; (2) while current cocrystal design strategies are aimed at identifying coformers based on the functional groups present on a given API, the design of cocrystals for molecules that lack potential hydrogen-bonding sites remains to be explored further. Such molecules are often prone to close packing via van der Waals interactions and there exists no reliable strategy for predicting cocrystal formation. A plausible solution to address this issue is to use knowledge-based methods, which incorporate structural, geometrical, and chemical information pertaining to the
API and coformers. ${ }^{59}$ The recent developments in cocrystal research suggest reliable pathways to identify suitable solid forms for development of novel drug formulations. In this regard, crystal structures deposited in the CSD serve as valuable source of information for the design of functional cocrystals. Over the past century, the role of X-ray crystallography as a tool to probe the structure and function of materials has greatly been acknowledged. Prediction of properties of a material based solely on its crystal structure is an emerging area of research and efforts in this direction further underscore the importance of $\mathrm{X}$-ray crystallography.

\section{Acknowledgements}

S. Aitipamula gratefully acknowledges the financial support from the Institute of Chemical and Engineering Sciences of $A^{\star}$ STAR (Agency for Science, Technology and Research), Singapore. V. R. Vangala thanks the Royal Society of Chemistry for Researcher Mobility Grant (2015/17).

Received: 14 January 2017 Accepted: 7 February 2017 Published online: 29 May 2017

\section{Open Access:}

This article is distributed under the terms of the Creative Commons Attribution 4.0 International License (http://creativecommons.org/ licenses/by/4.0/), which permits unrestricted use, distribution, and reproduction in any medium, provided you give appropriate credit to the original author(s) and the source, provide a link to the Creative Commons license, and indicate if changes were made.

\section{References}

1. Authier A (2013) Early days of X-ray crystallography, Oxford

2. Bragg WL (1949) The Crystalline state: a general survey, London

3. Kojić-Prodić B (2015) A century of X-ray crystallography and 2014 international year. Maced J Chem Chem Eng 34:19

4. Deschamps JR (2005) The role of crystallography in drug design. AAPS J 7:E813

5. Röntgen WC (1895) Phys Med Ges Würzburg 137:132

6. Friedrich W, Knipping P, Laue M (1912) InterferenzErscheinungen bei Röntgenstrahlen. Sitzungsber K Bayer Akad Wiss Muenchen 303-322

7. Bragg WH (1915) Bakerian lecture: X-rays and crystal structure Philos. Philos Trans R Soc Lond A 215:253

8. Desiraju GR (2014) Celebrating the International Year of Crystallography 2014. Cryst Growth Des 14:1 
9. Bragg WH, Bragg WL (1913) The structure of the diamond. Proc R Soc Lond A 89:277

10. He J, Kunitake T (2006) Are ceramic nanofilms a soft matter?. Soft Matter 2:119

11. Barnes WH (1929) The crystal structure of ice between 0 degrees C and - 183 degrees C. Proc R Soc Lond A 125:670

12. Hodgkin DC, Bernal JD (1934) X-ray photographs of crystalline pepsin. Nature 133:794

13. Kendrew JC, Bodo G, Dintzis HM, Parrish RG, Wyckoff H, Phillips DC (1958) A three-dimensional model of the myoglobin molecule obtained by X-ray analysis. Nature 181:662

14. Perutz MF, Rossmann MG, Cullis AF, Muirhead H, Will G, North AC (1960) Structure of haemoglobin: a threedimensional Fourier synthesis at 5.5-A. resolution, obtained by X-ray analysis. Nature 185:416

15. Hodgkin DC, Bunn CW, Rogers-Low BW, Turner-Jones A (1949) The X-ray crystallographic investigation of the structure of penicillin. Oxford University Press, Oxford

16. Brink C, Hodgkin DC, Lindsey J, Pickworth J, Robertson JH, White JG (1954) X-ray crystallographic evidence on the structure of vitamin B12. Nature 174:1169

17. Milton RC, Milton SCF, Kent BBH (1992) Total chemical synthesis of a D-enzyme: the enantiomers of HIV-1 protease show reciprocal chiral substrate specificity. Science 256:1445

18. Watson JD, Crick FHC (1953) Molecular structure of nucleic acids: a structure for deoxyribose nucleic acid. Nature 171:737

19. Groom CR, Bruno IJ, Lightfoot MP, Ward SC (2016) The Cambridge Structural Database. Acta Cryst B72:171

20. Berman HM, Westbrook J, Feng Z, Gilliland G, Bhat TN, Weissig H, Shindyalov IN, Bourne PE (2000) The protein data bank. Nucleic Acids Res 28:235

21. Cheetham AK, Goodwin AL (2014) Crystallography with powders. Nat Mat 13:760

22. Das R, Ali MdE, Hamid SBA (2014) Rev Adv Mater Sci 38:95

23. Debye P, Scherrer P (1916) Interferenzen an regellos orientierten Teilchen im Röntgenlicht. I. Phys Zeit 17:277

24. Hull AW (1917) A new method of X-ray crystal analysis. Phys Rev B 10:661

25. Attfield JP, Sleight AW, Cheetham AK (1986) Structure determination of $\mathrm{a}-\mathrm{CrPO}_{4}$ from powder synchrotron $\mathrm{X}$-ray data. Nature 322:620

26. Cheung EY, Kitchin SJ, Harris KDM, Imai Y, Tajima N, Kuroda R (2003) Direct structure determination of a multicomponent molecular crystal prepared by a solidstate grinding procedure. J Am Chem Soc 125:14658

27. Brittain HG (2001) X-ray diffraction III. Pharmaceutical applications of X-ray powder diffraction. Spectroscopy $16: 14$

28. Duggirala NK, Perry ML, Almarsson Ö, Zaworotko MJ (2016) Pharmaceutical cocrystals: along the path to improved medicines. Chem Commun 52:640
29. Byrn SR, Pfeiffer RR, Stowell JG (1999) Solid-state chemistry of drugs, 2nd edn. SSCI Inc, West Lafayette, Indiana

30. Bolla G, Nangia A (2016) Pharmaceutical cocrystals: walking the talk. Chem Commun 52:8342

31. Mestre-Ferrandiz JM, Sussex J, Towse A (2012) The R\&D cost of a new medicine. Office of Health Economics, London

32. Chong CR, Sullivan DJ (2007) New uses for old drugs. Nature 448:645

33. Khadka P, Ro J, Kim H, Kim I, Kim JT, Kim H, Cho JM, Yun G, Lee J (2014) Pharmaceutical particle technologies: An approach to improve drug solubility, dissolution and bioavailability. Asian J Pharm Sci 9:304

34. Puddipeddi M, Serajuddin ATM, Grant DJW, Stahl PH (2002) In: Stahl PH, Wermuth CG (eds) Handbook of pharmaceutical salts: properties, selection, and use. Wiley, Weinheim, pp 19-38

35. Generally Regarded As Safe chemicals by the US-FDA. http://www.fda.gov/Food/IngredientsPackagingLabeling/ FoodAdditivesIngredients/ucm091048.htm

36. Trask AV (2007) An overview of pharmaceutical cocrystals as intellectual property. Mol Pharm 4:301

37. Guidance for Industry: Regulatory Classification of Pharmaceutical Co-crystals; Food and Drug Administration: Silver Spring, MD, December 2011

38. EMA Reflection Paper on the Use of Cocrystals and other Solid State Forms of Active Substances in Medicinal Products. European Medicines Agency. 2014

39. http://www.fda.gov/downloads/drugs/guidancecomplianceregulatoryinformation/guidances/ucm516813.pdf

40. Aitipamula S, Banerjee R, Bansal AK, Biradha K, Cheney ML, Choudhury AR, Desiraju GR, Dikundwar AG, Dubey R, Duggirala N, Ghogale PP, Ghosh S, Goswami PK, Goud NR, Jetti RKR, Karpinski P, Kaushik P, Kumar D, Kumar V, Moulton B, Mukherjee A, Mukherjee G, Myerson AS, Puri V, Ramanan A, Rajamannar T, Reddy CM, Rodriguez-Hornedo N, Rogers RD, Row TNG, Sanphui P, Shan N, Shete G, Singh A, Sun CC, Swift JA, Thaimattam R, Thakur TS, Kumar Thaper R, Thomas SP, Tothadi S, Vangala VR, Variankaval N, Vishweshwar P, Weyna DR, Zaworotko MJ (2012) Polymorphs, salts, and cocrystals: what's in a name?. Cryst Growth Des 12:2147

41. Desiraju GR (1995) Supramolecular synthons in crystal engineering - a new organic synthesis. Angew Chem Int Ed Engl 34:2311

42. Etter MC (1990) Encoding and decoding hydrogen-bond patterns of organic compounds. Acc Chem Res 23:120

43. Desiraju GR (1989) Crystal engineering: the design of organic solids, Elsevier

44. Sanphui P, Devi VK, Clara D, Malviya N, Ganguly S, Desiraju GR (2012) Cocrystals of hydrochlorothiazide: solubility and diffusion/permeability enhancements through drug coformer interactions. Mol Pharm 12:1615

45. Bolton O, Matzger AJ (2011) Improved stability and smart-material functionality realized in an energetic cocrystal. Angew Chem Int Ed Engl 50:8960 
46. D'silva ED, Podagatlapalli GK, Rao SV, Rao DN, Dharmaprakash SM, (2011) New high efficiency nonlinear optical chalcone co-crystal and structure-property relationship. Cryst Growth Des 11:5362

47. Goud NR, Khan RA, Nangia A (2014) Modulating the solubility of sulfacetamide by means of cocrystals. Cryst Eng Comm 16:5859

48. Gopi SP, Banik M, Desiraju GR (2017) Cryst Growth Des $17: 308$

49. Toth PE, Urtis J (2004) Commonly used muscle relaxant therapies for acute low back pain: a review of carisoprodol, cyclobenzaprine hydrochloride, and metaxaloone. Clin Ther 26:1355

50. Aitipamula S, Chow PS, Tan RBH (2011) Conformational polymorphs of a muscle relaxant, metaxalone. Cryst Growth Des 11:4101

51. Holland J, Frampton C, Chorlton A, Gooding D (2014) Metaxalone cocrystals, US Pat. 8,871,793 B2

52. Vangala VR, Chow PS, Tan RBH (2011) Characterization, physicochemical and photo photostability of a cocrystal involving an antibiotic drug, nitrofurantoin, and 4- hydroxybenzoic acid. Cryst Eng Comm 13:759

53. Vangala VR, Chow PS, Tan RBH (2012) Co-crystals and co-crystal hydrates of the antibiotic nitrofurantoin: structural studies and physicochemical properties. Cryst Growth Des 12:5925
54. Karki S, Friščić T, Fábián L, Laity PR, Day GM, Jones W (2009) Improving mechanical properties of crystalline solids by cocrystal formation: new compressible forms of paracetamol. Adv Mater 21:3905

55. Ahmed H, Shimpi MR, Velaga SP (2016) Relationship between mechanical properties and crystal structure in cocrystals and salt of paracetamol. Drug Devel Ind Pharm. doi:10.1080/03639045.2016.1220568

56. FDA Approves New Drug to Treat Heart Failure. http:// www.fda.gov/NewsEvents/Newsroom/PressAnnouncements/ucm453845.htm

57. Ranzani LS, Aldea AF (2013) Pharmaceutical compositions of co-crystals of tramadol and coxibs, US Pat. 2013/0109659 A1

58. Bernhardson D, Brandt TA, Hulford CA, Lehner RS, Preston BR, Price K, Sagal JF, St. Pierre MJ, Thompson PH, Thuma B (2014) Development of an early-phase bulk enabling route to sodium-dependent glucose cotransporter 2 inhibitor ertugliflozin. Org Process Res Dev 18:57

59. Mapp LK, Coles SJ, Aitipamula S (2017) Design of cocrystals for molecules with limited hydrogen bonding functionalities: propyphenazone as a model system. Cryst Growth Des 17:163

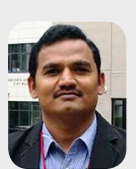

Srinivasulu Aitipamula is a Scientist in Crystallization and Particle Sciences Division at the Institute of Chemical and Engineering Sciences (ICES) of $\mathrm{A}^{\star} \mathrm{STAR}$ (Agency for Science, Technology and Research), Singapore. He obtained his Ph.D. in 2006 from the University of Hyderabad (India) working with Prof. Ashwini Nangia on structural and thermochemical studies of some host-guest systems and polymorphs. After a short industrial stint as a Manager-R\&D at Shasun Research Centre, India, he joined ICES in 2007. He has nearly 50 peer-reviewed publications including two book chapters. He is currently the guest editor for a special issue on "Novel pharmaceutical cocrystals and their applications" in the journal Crystals. His research interests include crystal engineering, solid-form screening of active pharmaceutical ingredients, and formulation development.

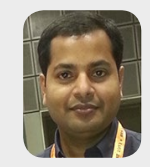

Venu R. Vangala is a Lecturer in Medicines Development and Pharmaceutical Science at School of Pharmacy and Medical Sciences, University of Bradford, UK. He completed M.Sc. from Kakatiya University, Warangal (1998) and Ph.D. in crystal engineering (Mentor: Prof. Gautam R. Desiraju) from the School of Chemistry, University of Hyderabad, Hyderabad (2004). Subsequently, he did a brief industrial stint as Research Associate at GVK Biosciences, Hyderabad (2004-2005) and did post-doctoral research at the University of Iowa, USA (2005-2007) and University of Minnesota, USA (2007-2009), and worked as Scientist at ICES of A*STAR, Singapore (2009-2014). He is with the University of Bradford since July 2014 . He is the author of nearly 30 publications. His current research is focused on crystal engineering for pharmaceuticals and nutraceuticals development, solid forms pre-formulations (cocrystals, polymorphs, salts, and amorphous materials), crystal structure and pharmaceutical, physicochemical, and mechanical property understanding, and formulations. http://bradford.ac.uk/life-sciences/pharmacy/our-staff/ venu-vangala.php. 